\title{
Influence of short-term ovarian stimulation on bone metabolism in women undergoing fertility treatment
}

\author{
https://doi.org/10.1515/labmed-2020-0117 \\ Received October 1, 2020; accepted January 12, 2021; \\ published online February 4, 2021
}

\begin{abstract}
Objectives: Hormonal disturbances during menopause are an established influencing factor on bone health, but the role of controlled ovarian hyperstimulation for fertility treatment remains unclear. To evaluate the influence of ovarian stimulation on bone metabolism with particular regard to serum follicle-stimulating hormone (FSH) levels this prospective observational study was conducted.

Methods: A total of 71 women underwent controlled ovarian hyperstimulation with recombinant FSH ( $\mathrm{rFSH})$ or human menopausal gonadotropin (HMG) (FSH + LH) administered in individual doses, with gonadotropinreleasing hormone ( $\mathrm{GnRH}$ ) agonist down-regulation initiated in the luteal phase of the previous cycle. At four time points (start of down-regulation [T1], start of ovarian stimulation [T2], oocyte retrieval [T3] and luteal phase of the stimulation cycle [T4]), luteinizing hormone (LH), FSH, estradiol (E2), osteocalcin (OC), bone-specific alkaline phosphatase (BAP), as well as the bone resorption markers $\beta$-isomerized C-terminal telopeptide of type I collagen ( $\beta$-CTX) and tartrate-resistant acid phosphatase (TRACP) were measured.
\end{abstract}

Results: The cyclic variations in FSH levels had a positive effect on the concentration profile of the bone resorption marker $\beta$-CTX $(p=0.0001)$. Supraphysiologic estradiol

Anna Ritzinger and Monika Schmidmayr contributed equally as first authors.

*Corresponding author: Dr. Monika Schmidmayr, Reproductive Endocrinology, Klinik und Poliklinik für Frauenheilkunde, Technische Universität München (TUM), Ismaninger Str. 22, 81675 Munich, Germany, Phone: +49 8941405434 , E-mail: Monika.Schmidmayr@tum.de Anna Ritzinger and Vanadin Seifert-Klauss, Reproductive Endocrinology, Klinik und Poliklinik für Frauenheilkunde, Technische Universität München (TUM), Munich, Germany

Bernd Lesoine, A.R.T. Bogenhausen, Munich, Germany levels showed a negative association with osteocalcin concentrations $(\mathrm{p}=0.017)$, and significantly lower OC and TRACP levels were observed at T4 compared to T1. By group comparison, women treated with $\mathrm{rFSH}$ presented with a higher bone turnover than the HMG group at the end of a stimulation cycle (T4).

Conclusions: Our results show that FSH is a significant influencing factor of bone metabolism. Overall, there was no evidence of enhanced bone resorption under short-term ovarian stimulation therapy. Further studies with bigger sample sizes are warranted to validate these results.

Keywords: bone metabolism; osteoporosis; ovarian stimulation; sterility.

\section{Introduction}

The osteoprotective effect of estradiol on bone metabolism is well established. However, the fact that during perimenopause, there is substantial bone loss despite normal-range or even elevated estrogen levels has led to the hypothesis that other hormones might play a role [1]. In 2006, Sun et al. reported that hypogonadal FSH receptor null mice displayed no loss in bone density. Beyond that, their study showed that follicle-stimulating hormone (FSH) simulates osteoclast formation by activating osteoclast-bound FSH receptors [2]. The question thus arises whether the rising FSH levels in perimenopause may be responsible for an activated bone turnover during this period.

Osteoporosis is one of the 10 most common diseases worldwide [3], and according to Johnell and Kanis, the global burden of osteoporotic fractures in terms of mortality and the impairment in quality of life as quantified by DALYs (disability-adjusted life years) is greater than e.g. the burden of colorectal or breast cancer [4].

Bone changes during perimenopause happen in an endocrinological transition phase characterized by elevated gonadotropins in the presence of still adequate estradiol serum levels. Isolated elevations of follicle 
stimulating hormone (FSH) in the early follicular phase of the cycle are the first and early biochemical hallmark of ovarian aging, as known from reproductive medicine. Later in the transition process, luteinizing hormone (LH) also rises, and both gonadotropins remain elevated for around seven years before declining to their postmenopausal resting state.

One situation which resembles perimenopause in a short-term model, is the controlled ovarian stimulation used for in vitro fertilization (IVF) treatments. At the beginning of a natural cycle FSH is stimulating the growth of one follicle which is producing estradiol, so around ovulation serum estradiol levels reach a level of around $300 \mathrm{pg} / \mathrm{ml}$. After ovulation estradiol and progesterone are produced by the corpus luteum, and the gonadotropins are at a low level. At the end of the cycle FSH is rising again in order to recruit follicles for the next cycle. In the long protocol of in vitro fertilization, the natural female cycle is initially down-regulated for 10-12 days in the luteal phase of the previous cycle by applying gonadotropin-releasing hormone ( $\mathrm{GnRH})$ analogues. Then oocyte maturation is stimulated by administering recombinant $\mathrm{FSH}(\mathrm{rFSH})$ alone or $\mathrm{rFSH}$ in combination with LH (in the form of human menopausal gonadotropin [HMG]) in very much higher doses than during the natural cycle, which results in 5-10 mature follicles producing estradiol serum levels up to $3,000 \mathrm{pg} /$ $\mathrm{mL}$. This leads to elevated gonadotropin and elevated estradiol levels at the same time, a situation like perimenopause.

In this study, bone formation was studied using bone-specific alkaline phosphatase (BAP), an enzyme produced by osteoblasts, as well as osteocalcin (OC), a small, non-collagenous protein which is also synthesized by osteoblasts [5]. Bone resorption was reflected by serum $\beta$-isomerized $C$-terminal telopeptide of type I collagen ( $\beta$-CTX), a fragment produced from collagen chains as a result of osteoclast resorption activity, and tartrate-resistant acid phosphatase 5b (TRACP), a lysosomal enzyme released by osteoclasts [5]. As bone formation and bone resoprtion are coupled, all parameters eventually reflect bone turnover [6].

The controlled modeling of menstrual cycle hormone profiles during the course of ovarian stimulation for IVF treatment was used to study the short-term effects of the administered hormones on bone metabolism. In the present study, we also compared rFSH and HMG stimulation regimens as models for early (FSH-elevation) and later (FSH- and LH-elevation) perimenopause.

The hypothesis in this study was whether and which changes in parameters of bone metabolism can be seen during an IVF cycle.

\section{Materials and methods}

\section{Study population and study design}

Between September 2012 and February 2013, patients were recruited from the IVF center collaborating with the outpatient department for reproductive medicine of the Klinik und Poliklinik für Frauenheilkunde of TUM (University Gynecological Clinic). Women aged 30-45 years with a baseline serum FSH level of $<20 \mathrm{mIU} / \mathrm{mL}$ were eligible for the study. Individuals with conditions or treatments affecting bone metabolism like daily smokers were excluded from the study. The study protocol was reviewed and approved by the Ethics Committee of the Technical University of Munich. Patient data were collected after written informed consent was obtained. Baseline demographics of the study population are shown in Table 1.

Buserelin, nafarelin or triptorelin were used for pituitary downregulation. HMG-treated patients were treated with human urinary FSH $+\mathrm{LH}$, in a ratio of 1:2 $\left(\right.$ Menogon $^{\circledR}$, Menopur $\left.^{\circledR}\right)$ in the majority of cases, less often Merional ${ }^{\circledR}$ (urinary human LH, FSH 1:2, and human chorionic gonadotropin [HCG]) were administered. Follitropin beta (Puregon ${ }^{\circledR}$ ) was used as recombinant FSH preparation. Ovulation was triggered with HCG or the GnRH agonist triptorelin. Blood samples for analyses of bone metabolism biomarkers and hormonal levels were drawn at four different time points (T1-T4) in the course of an IVF treatment cycle as shown in Figure 1: at the start of down-regulation in the luteal phase preceding the stimulation cycle (T1), at the start of ovarian stimulation with HMG or $\mathrm{rFSH}$ in the follicular phase of the stimulation cycle (T2), at the time of oocyte retrieval (T3) and in the luteal phase of the stimulation cycle (T4).

\section{Parameters of bone metabolism and hormonal levels}

FSH (Chemiluminescence-, Immunometric Assay, Siemens Healthcare Diagnostics, Eschborn, Germany), LH (Chemiluminescence-, Immunometric Assay, Siemens Healthcare Diagnostics, Eschborn, Germany) and estradiol (competitive Chemilumineszenz-Immunoassay, Siemens Healthcare Diagnostics, Eschborn, Germany) were assessed at all four time points and progesterone levels (competitive ChemiluminescenceImmunoassay, Siemens Healthcare Diagnostics, Eschborn, Germany) were measured at $\mathrm{T} 1$ and $\mathrm{T} 4$. In addition, the concentrations of $25(\mathrm{OH})$ vitamin D3 (CLIA, DiaSorin, Dietzenbach, Germany) and calcium (photometry test with NM-BAPTA, Roche, Penzberg, Germany) were determined at T1. The bone formation biomarkers BAP (DiaSorin, Dietzenbach, Germany) and OC (ECLIA, Roche Penzberg, Germany) as well as the bone resorption biomarkers TRACP (ELISA, Dynex, Lidicka, Czech Republic) and $\beta$-CTX (ECLIA, Roche, Penzberg, Germany) were

Table 1: Baseline demographics of the study population.

\begin{tabular}{lrrrrrr}
\hline & $\mathbf{n}$ & Minimum & Maximum & Median & Mean & SD $^{\mathbf{a}}$ \\
\hline Age at T1, years & 71 & 30 & 45 & 38 & 37.82 & 3.36 \\
25(OH)-vitamin D, & 71 & 5 & 47 & 18 & 19.56 & 9.05 \\
ng/mL & & & & & & \\
Calcium, mmol/L & 71 & 2.11 & 2.71 & 2.42 & 2.42 & 0.09 \\
BMI, kg/m & 71 & 18.65 & 42.52 & 21.67 & 22.92 & 4.43 \\
\hline
\end{tabular}

SD, standard deviation; BMI, body-mass-index. 
assessed at all four time points. OC and $\beta$-CTX were analyzed via electrochemiluminescence immunoassay (ECLIA), BAP via solid phasechemiluminescence immunoassay (CLIA), TRACP via enzyme-linked immunosorbent assay (ELISA). Blood samples were drawn in the morning hours before noon in order to prevent fluctuations in concentrations due to circadian rhythms. Blood samples that were not analyzed immediately were quickly aliquoted, stored frozen at $-20^{\circ} \mathrm{C}$ and protected from heat and light during transportation. Multiple freeze-thaw cycles were avoided.

\section{Statistical methods of analysis}

The data were assessed for distribution. Hormonal and bone parameters at the respective time points were compared by paired samples t-test, and additionally (for the analysis of $\mathrm{rFSH}$ vs. HMG stimulation) by unpaired samples t-test.

As the intention was to not only compare values at particular points in time, but also to evaluate the impact of the course of cyclic variations in hormone profiles on the parameters of bone metabolism, the regular Multiple Regression Model was insufficient. Intraindividual correlations bias the results and therefore had to be considered in the regression analysis.

This study comprised longitudinal data and it had to be assumed that the up to four observations of one patient were not independent of each other. Therefore, the linear regression was extended by a Repeated Measures Approach. This involved adding an individual effect for every patient as well as specifying the covariance matrix for the measurements of an individual person. Simply speaking, one model was calculated for every patient and the overall effect was calculated from the results of all patients.

Adding the factor time as a covariate to the model was a prerequisite to be able to correctly model possible developments which did not result from the other influencing variables, but from the course of time itself.

In order to avoid confounding between FSH and estradiol, the model was calculated once for both hormones together and for each hormone individually.

The statistics software programs R and SAS allow the estimation of this Repeated Measures Model with the use of different functions. In the presented study the procedure GENMOD was used in SAS.

\section{Results}

Of the 95 participants, 71 completed a full, long protocol IVF treatment cycle and were included in the bone marker analyses.

\section{Temporal profiles of hormonal and bone metabolic parameters under IVF treatment}

As shown in Figure $2 \mathrm{FSH}$ and estradiol displayed a similar pattern from T2 to T4 (luteal phase of the stimulation cycle) a steep increase from T2 (start of stimulation) to T3, followed by a sharp decline from T3 (oocyte retrieval) to T4. Median values of FSH were within the respective reference range, while estradiol levels reached supraphysiological median values of $1,138.5 \mathrm{pg} / \mathrm{mL}$ at T3 (reference range of estradiol levels during the periovulatory phase of natural cycles: up to $400 \mathrm{pg} / \mathrm{mL}$ ). Results of the paired samples t-test revealed that all changes in estradiol and FSH levels between the single time points were significant, with the exception of the delta between $\mathrm{T} 1$ (downregulation) and T2 for FSH.

Evaluation of the temporal profiles of bone metabolism biomarkers showed declining BAP, OC and TRACP levels from T1 over T2 to T3, as shown in Figure 3. However, concentrations of BAP and OC increased again between T3 and T4, while TRACP levels remained the same. $\beta$-CTX concentrations, on the contrary, rose from $\mathrm{T} 1$ (downregulation) to $\mathrm{T} 2$ (start of hyperstimulation) and to $\mathrm{T} 3$ (oocyte retrieval), then decreased again from $\mathrm{T} 3$ to $\mathrm{T} 4$. When comparing baseline values, i.e. at $\mathrm{T} 1$, with the end-oftreatment concentrations, i.e. at T4, OC and TRACP levels turned out to be significantly lower at $\mathrm{T} 4$, whereas BAP and $\beta$-CTX values were found to not be significantly different.

\section{Influence of hormones on biochemical bone parameters over time}

The bone formation biomarkers BAP and OC displayed an inverse temporal profile to FSH and estradiol, while the profile of the bone resorption biomarker $\beta$-CTX followed a similar course to FSH and estradiol curves. Results for TRACP were mixed, however, the course of its profile rather resembling that of bone formation parameters.

The association of estradiol with osteocalcin levels over time was found to be significantly negative in repeated measures analysis, regarding the association of estradiol on osteocalcin alone $(\mathrm{p}=0.0166)$ as well as when FSH was included in the model $(\mathrm{p}=0.0276)$. In contrast, FSH showed a positive correlation with the bone resorption biomarker $\beta$-CTX over time (model considering FSH and estradiol: $\mathrm{p}=0.0002$; model without estradiol: $\mathrm{p}=0.0001$, see Figure 4). Detailed values are shown in Table 2.

\section{Comparison of bone parameters and hormone levels under rFSH vs. HMG stimulation}

The patient population was subdivided into two groups according to the type of ovarian stimulation (stimulation with HMG: $\mathrm{n}$ at T3 $=54, \mathrm{n}$ at T4=45; stimulation with $\mathrm{rFSH}: \mathrm{n}$ at $\mathrm{T} 3=17, \mathrm{n}$ at $\mathrm{T} 4=13$ ). In the unpaired samples t-test, both groups did not differ significantly with respect to $25(\mathrm{OH})$ - 

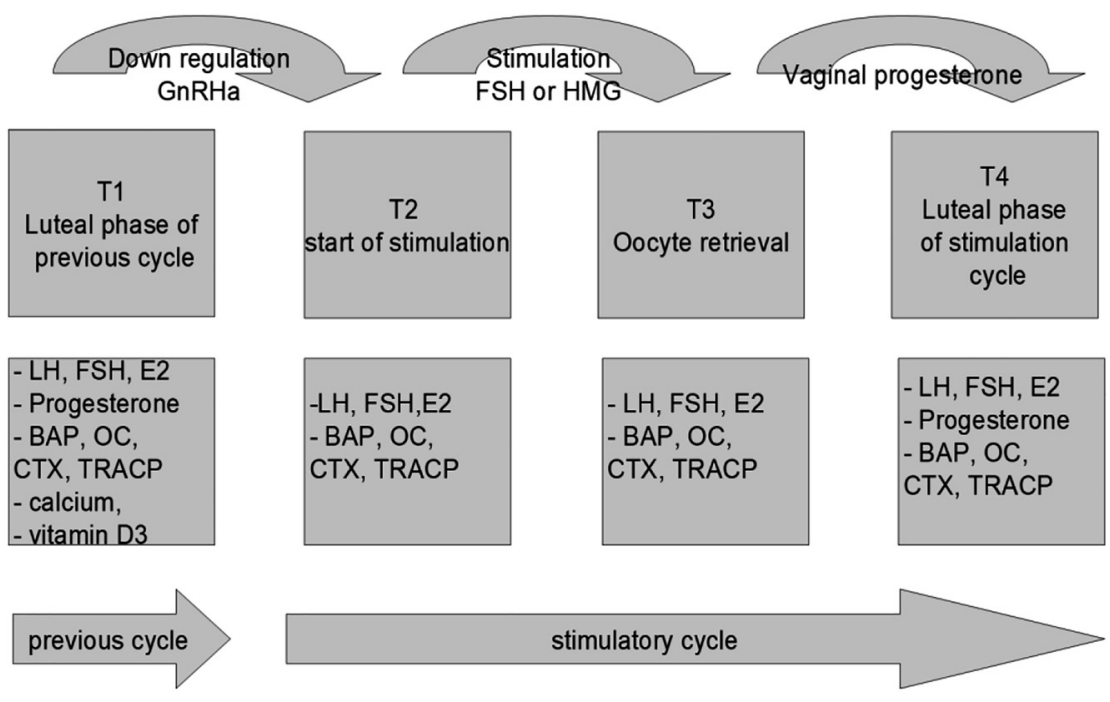

Figure 1: Study design.

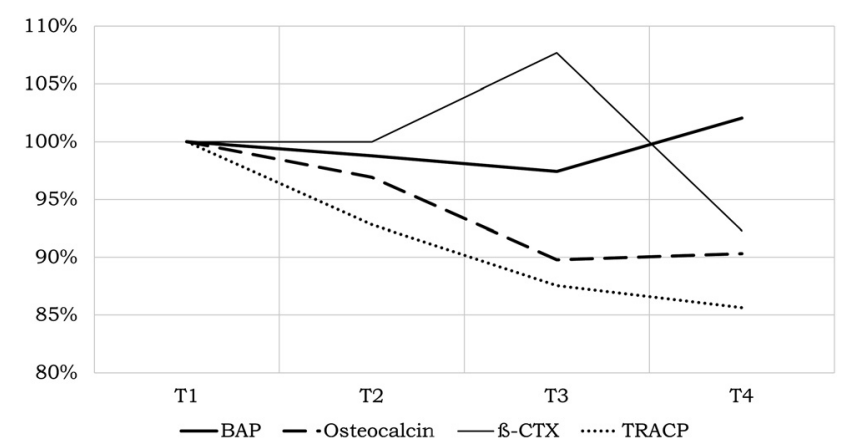

Figure 2: Comparison of the percentage change of the temporal profiles of BAP, OC, B-CTX and TRACP.

Time point 1 (T1), luteal phase of the previous cycle; time point 2 (T2), start of stimulation; time point 3 (T3), oocyte retrieval; time point 4 (T4), luteal phase of the stimulation cycle.

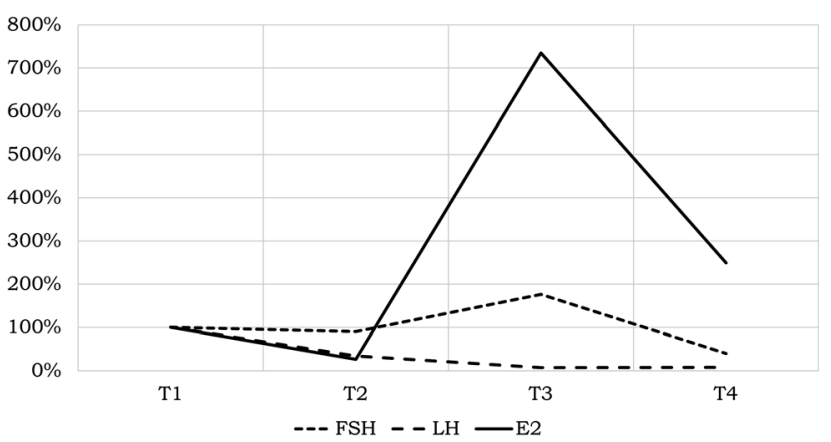

Figure 3: Comparison of the percentage change of the temporal profiles of FSH, LH and E2.

Time point 1 (T1), luteal phase of the previous cycle; time point 2 (T2), start of stimulation; time point 3 (T3), oocyte retrieval; time point 4 (T4), luteal phase of the stimulation cycle.

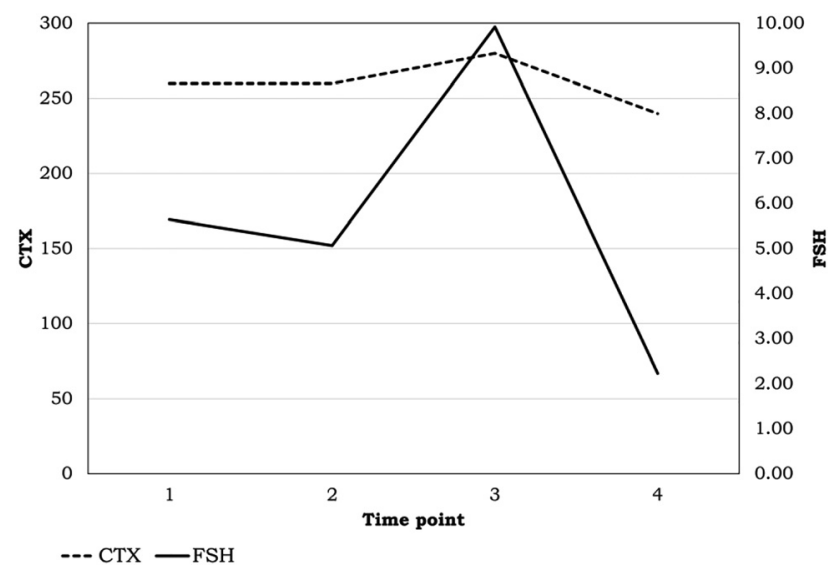

Figure 4: Comparison of the temporal profiles of $\mathrm{FSH}(\mathrm{mlU} / \mathrm{mL})$ and $\beta$-CTX (ng/L).

Time point 1 (T1), luteal phase of the previous cycle; time point 2 (T2), start of stimulation; time point 3 (T3), oocyte retrieval; time point 4 (T4), luteal phase of the stimulation cycle; a highly significant influence of FSH on $\beta$-CTX levels was noted $(p=0.0001)$.

vitamin D and calcium levels, BMI, ovulation frequency or age. Hormone levels and bone parameters in both groups were compared by unpaired samples t-test after the start of stimulation, at $\mathrm{T} 3$ and $\mathrm{T} 4$.

By group comparison, bone parameters showed no significant differences at both time points T3 and T4. At T4, however, slightly higher levels of bone markers were noted in patients treated with $\mathrm{rFSH}$ alone (Figure 5).

In addition, serum FSH concentrations at $\mathrm{T} 4$ were significantly higher when only $\mathrm{rFSH}$ was administered $(\mathrm{p}=0.044)$ than following stimulation with $\mathrm{HMG}$, while serum estradiol levels in the $\mathrm{rFSH}$ group were significantly lower at T4 (p=0.012) (Figure 6). 

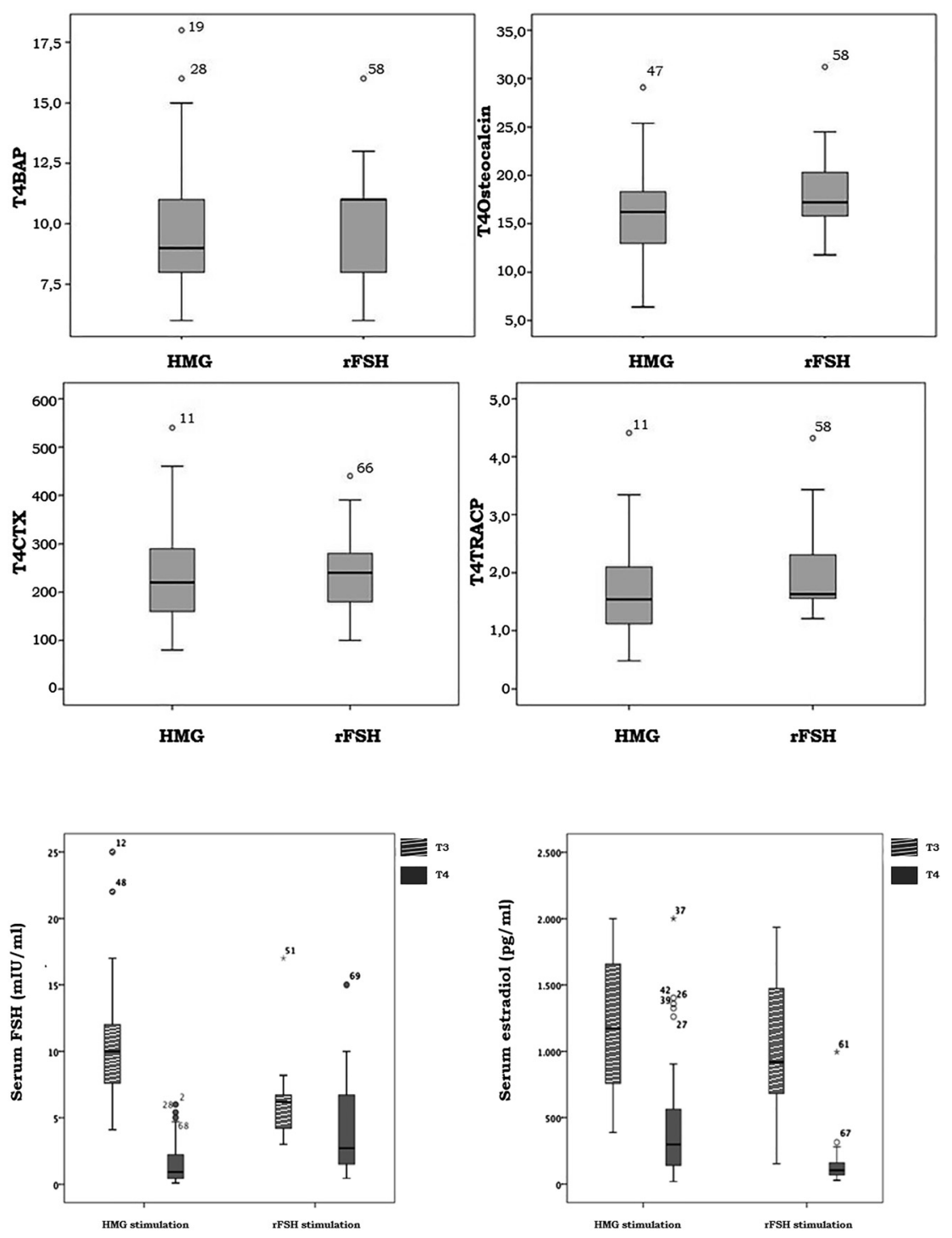

Figure 5: Comparison of the levels of bone metabolism parameters in rFSH vs. HMG stimulation at $\mathrm{T} 4$.

A trend towards higher concentrations of bone metabolism parameters was noted in the $r F S H$ treatment group (BAP: $p=0.692$; OC: $p=0.128 ; \beta-C T X: p=0.79$; TRACP: $p=0.152$ ).

\section{Discussion}

In the normal healthy skeleton, bone formation and resorption are coupled, so that osteoclastic bone resorption, lasting a few days, is followed by the slower process of bone formation, ultimately equaling the amount of bone which was previously resorbed [6]. This bone remodeling is reflected by the biochemical markers of bone metabolism, measurable in serum or urine [7]. We used therapeutic ovarian stimulation as a model to study and possibly dissect the influence of FSH from that of estradiol on bone metabolism, as high FSH in postmenopause is generally accompanied by low estradiol, which makes it difficult to differentiate the respective effects.

Hormone levels during the ovarian stimulation treatment cycles in this study showed the expected temporal profiles, with FSH and estradiol concentrations significantly increasing between $\mathrm{T} 2$ and $\mathrm{T} 3$ with growing follicles under the influence of exogenously administered FSH. As anticipated, the concentrations of both hormones dropped in the luteal phase and after exogenous FSH administration was stopped. As intended, LH secretion and the physiological preovulatory LH surge were suppressed by administration of GnRH agonists.

\section{Effect of FSH on the skeleton}

The osteoprotective effect of estradiol is well-known and well-established. In this study, the protective effect of estradiol on bone was reflected by the inverse temporal profiles of estradiol and osteocalcin, with the repeated 
Table 2: Detailed values of hormones and bone parameters from T1 to $\mathrm{T} 4$.

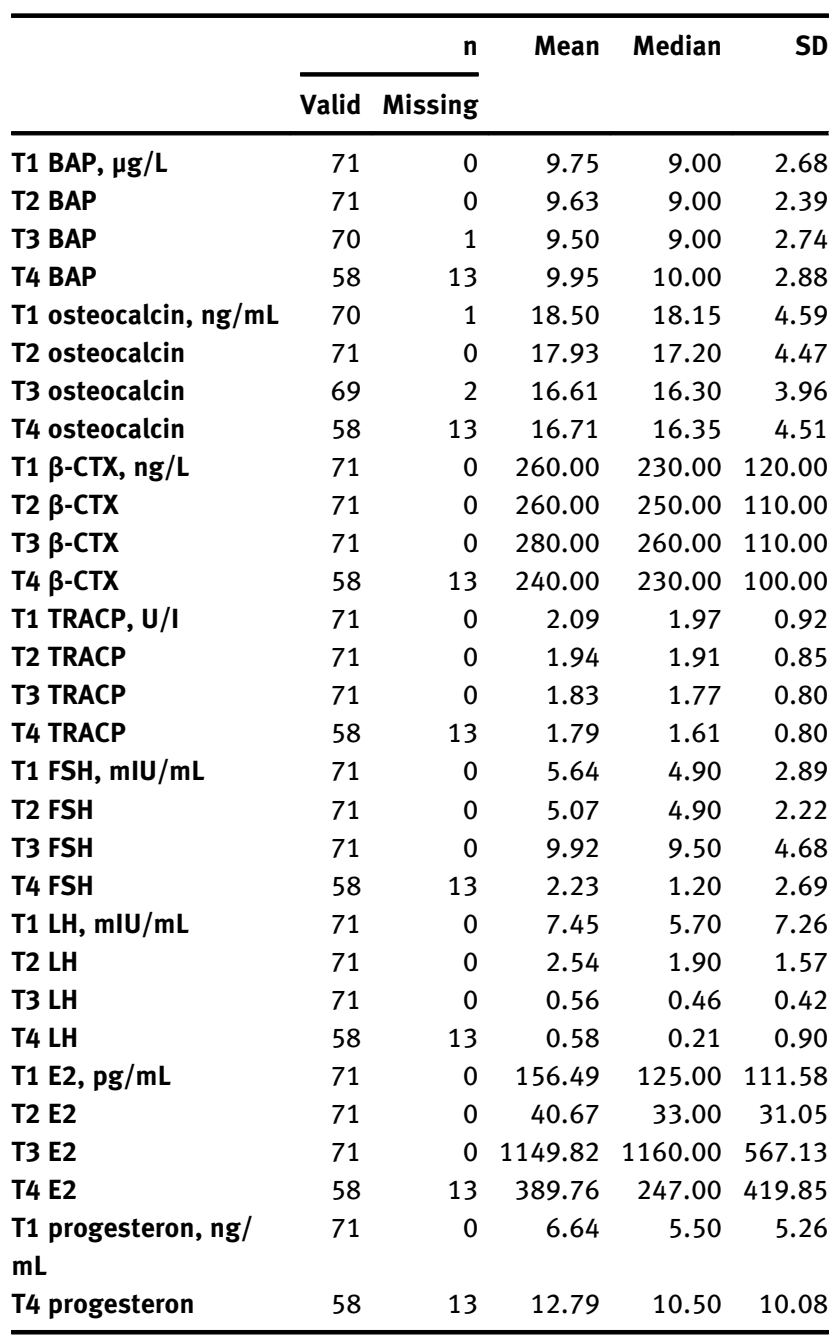

SD, standard deviation; BAP, bone-specific alkaline phosphatase; $\beta$-CTX, $\beta$-isomerized $C$-terminal telopeptide of type I collagen; TRACP, tartrate-resistant acid phosphatase; $\mathrm{FSH}$, follicle-stimulating hormone; LH, luteinizing hormone; E2, estradiol.

measures model showing a significantly negative correlation between estradiol and osteocalcin over time, corresponding to a down-regulation of bone turnover.

Women in the perimenopausal phase experience an accelerated loss in bone density despite high estradiol levels and concurrent high concentrations of $\mathrm{FSH}$, and this is one of the reasons why the question of whether elevated serum FSH levels negatively affect bone metabolism is controversially discussed [1]. In 2006, Sun et al. published the results of their study on FSH receptor knockout mice which - in contrast to ovariectomized mice with intact FSH receptors - showed no loss in bone density despite severe hypogonadism. These results led to the conclusion that FSH may enhance osteoclast formation and function through various different pathways [2]. The same research group was able to demonstrate that FSH stimulates TNF-alpha production, thus increasing bone resorption [8]. The suitability of FSH receptor knockout mice is critically discussed, partly because testosterone concentrations are high and estradiol levels are reduced in these mice, with corresponding bone-related effects $[9,10]$. Zhu et al. solved this problem by using ovariectomized wild-type mice showing a marked loss in bone volume fraction (BV/TV) ratio due to the resulting severe hypogonadism, and subsequently injecting them with an anti-FSH receptor antibody. The anti-FSH receptor antibody induced an increase in BV/TV, however, could not fully compensate the BV/TV loss caused by the hypogonadic state [10]. The modulation of gonadotropin-hormone-activity by using therapeutic antibodies might provide further opportunities in studying the influence of these hormones on bone in the future. Kara et al. even proposed antibodies to be appropriate in treating bone loss potentially caused by high FSH by blocking the effect of FSH [11].

In our study, the cyclic changes of FSH showed a significant positive association with the bone resorption biomarker $\beta$-CTX: increased FSH levels corresponded with elevated concentrations of $\beta$-CTX and decreased FSH levels with reduced $\beta$-CTX concentrations. This is supportive of the argument that FSH may promote bone resorption $[2,8$, $12,13]$. As early as in 2006, Sun et al. have noted that an association between FSH and the parameters of bone resorption is also observed in natural menstrual cycles [2]. Thus, in natural menstrual cycles levels of bone resorption biomarkers, in particular $\beta$-CTX, increase during the early to mid-follicular period and then continuously drop until the end of the luteal phase after which they rise again relatively quickly [14-16]. This most closely corresponds to the natural secretion pattern of FSH, which rises steeply before the beginning of the follicular phase and continuously falls thereafter, apart from a slight peak around ovulation time $[17,18]$.

By contrast, Omodei et al. reported no significant change of $\beta$-CTX during stimulation with FSH (day 10). That group had followed 29 women from the start of down-regulation (T0, as in the present study), through the beginning of FSH-apllication, day three and 10. They found serum $\beta$-CTX levels to be significantly lower at day 10 compared to the day of FSH-initiation (10-12 days after $\mathrm{GnRH}$ ) and concluded that not FSH but primarily estradiol affected bone [19]. However, both FSH and estradiol rose between stimulation-day three and 10 from physiological to supraphysiological levels, so a differential effect of FSH could not really be proven. The results of the present study support the observation of concordant temporal profiles of $\beta$-CTX and FSH and therefore support the 
assumption that FSH promotes bone resorption independent of low estradiol.

\section{Effect of the type of ovarian stimulation on bone metabolism}

After ovarian stimulation was initiated, significant differences in serum hormone levels were observed between the two groups treated with rFSH or HMG.

Patients stimulated with $\mathrm{rFSH}$ showed significantly lower serum FSH levels at T3 $(\mathrm{p}=0.0001)$ and significantly higher serum FSH levels at T4 $(\mathrm{p}=0.044)$, compared to those treated with HMG. One possible explanation for both observations is that some patients participating in this study were PCOS patients. PCOS patients are preferably treated with pure rFSH for controlled hyperstimulation and feature both lower estradiol and lower FSH compared with LH in their natural cycles, which explains lower FSH at T3 in this group. Also, PCOS itself may have unfavourable implications for bone metabolism due to chronic low-grade inflammation, as suggested in 2017 by Kalyan et al. [18].

At the time of oocyte retrieval (T3), there were no significant differences in mean serum estradiol levels with respect to the type of ovarian stimulation treatment (owing to the exogenous stimulation), whereas in the luteal phase of the stimulation cycle (T4), mean serum estradiol levels were significantly lower in women stimulated with $\mathrm{rFSH}$ ( $\mathrm{p}=0.012)$. Similarly, no significant difference in bone markers was observed at T3, while at T4, all parameters of bone metabolism displayed slightly higher values (n.s.) when $\mathrm{rFSH}$ had been administered alone.

These tendencies towards a higher bone turnover after the stimulation of $\mathrm{rFSH}$ at $\mathrm{T} 4$ could be attributed both to the higher serum FSH concentrations and the lower serum estradiol levels. Whether the influence of serum estradiol dominates over that of FSH, or whether the possible influence of inflammatory processes in PCOS-bone metabolism plays a role [18], awaits further study. Overall, we observed no significant bone turnover-differences between the two stimulation groups, however, at $\mathrm{T} 4$, there was a trend towards a higher bone turnover in the rFSH group, in the presence of both higher serum FSH and lower serum estradiol levels. The fact that statistical significance was missed is possibly due to the small sample size.

\section{Study limitations}

The influence of circadian rhythms on the levels of bone metabolism biomarkers was adressed by drawing blood samples in the mornings before noon. $\beta$-CTX in particular shows a circadian rhythm with a peak in the early morning hours (around 2-6 a.m.) and a nadir in the early afternoon (around 12-3 p.m.) [19-21]. Blood samples were therefore drawn between 8 and 11 a.m. however not every patient was fasting in advance. Occasional afternoon values during the study did not influence the main results, as analyses which excluded them showed.

A further limitation of this study is the small sample size. Although the total sample size was more than twice the size of the Omodei-paper published in JCEM, numbers were further diminished in the subgroups to investigate the influence of the type of ovarian stimulation on bone turnover. Still, the HMG-stimulated group even then comprised 45 participants, whereas the rFSH group was small with 13 participants at $\mathrm{T} 4$.

Finally, this study only observed one month duration. Further research would be needed to judge the effect of several consecutive cycles.

\section{Conclusions}

The cyclic variation pattern of FSH showed a significantly positive influence on the temporal profile of the bone resorption biomarker $\beta$-CTX ( $\mathrm{p}=0.0001)$ which points out that FSH levels negatively affect bone metabolism. This corresponds to the postulate of Sun et al. [2] according to which FSH exerts a direct effect on bone resorption.

The short-term influence of $\mathrm{rFSH}$ or FSH/LH administration on bone metabolism during fertility treatment was reflected by suppressed mean levels of TRACP (delta T1/T4, $\mathrm{p}=0.0001$ ), OC (delta T1/T4, $\mathrm{p}=0.006$ ) at T4, likely due both to the strongly elevated estradiol concentrations and to the suppressed endogenous FSH (and LH) levels during the treatment cycle. Overall, ovarian stimulation treatment for one cycle seems to have a rather transient osteo-metabolic effect and there does not seem to be an increased risk of bone resorption.

The question as to whether the type of ovarian stimulation treatment (LH/FSH vs. $\mathrm{rFSH}$ ) has an influence on bone metabolism could not be fully answered because sample sizes were small. There was no significant difference of bone markers between both groups. At the end of the stimulation cycle (T4), all parameters of bone metabolism tended to be higher in the group stimulated with $\mathrm{rFSH}$, in the presence of significantly higher serum FSH levels and significantly lower serum estradiol levels than in the HMG group. These observations could be in line with our finding that FSH promotes bone resorption. However it has to be proven whether the higher number of 
PCOS-patients suffering chronic low-grade inflammation may be the reason for higher bone turnover in the rFSH-group.

For a final assessment of the effects of ovarian stimulation on bone health further research with bigger sample sizes is needed.

\section{Highlights}

- FSH is an influencing factor on parameters of bone metabolism.

- Short term rFSH/HMG-treatment does not significantly enhance parameters of bone resorption.

- No significant difference between $\mathrm{rFSH}^{-}$und HMG-stimulation has been found.

Research funding: Ferring $\mathrm{GmbH}$.

Author contributions: All authors have accepted responsibility for the entire content of this manuscript and approved its submission.

Competing interests: Authors state no conflict of interest. Informed consent: Informed consent was obtained from all individuals included in this study.

Ethical approval: The study protocol was reviewed and approved by the Ethics Committee of the Technical University of Munich.

\section{References}

1. Seifert-Klauss V, Link T, Heumann C, Luppa P, Haseitl M, Laakmann J, et al. Influence of pattern of menopausal transition on the amount of trabecular bone loss. Results from a six-year prospective longitudinal study. Maturitas 2006;55:317-24.

2. Sun L, Peng Y, Sharrow AC, Iqbal J, Zhang Z, Papachristou DJ, et al. FSH directly regulates bone mass. Cell 2006;125:247-60.

3. Bartl R, editor. Klinische Osteologie. Stuttgart: Georg Thieme Verlag KG; 2014.

4. Johnell O, Kanis JA. An estimate of the worldwide prevalence and disability associated with osteoporotic fractures. Osteoporos Int 2006;17:1726-33.

5. Hlaing TT, Compston JE. Biochemical markers of bone turnover uses and limitations. Ann Clin Biochem 2014;51:189-202.

6. Baron R. Anatomy and ultrastructure of bone-histogenesis, growth and remodeling. In: De Groot LJ, Beck-Peccoz P, Chrousos G, Dungan K, Grossman A, Hershman JM, et al, editors. Endotext. South Dartmouth, MA: MDText.com, Inc.; 2000.
7. Seibel MJ. Clinical application of biochemical markers of bone turnover. Arquivos Brasileiros Endocrinol Metabol 2006;50: 603-20.

8. Iqbal J, Sun L, Kumar TR, Blair HC, Zaidi M. Follicle-stimulating hormone stimulates TNF production from immune cells to enhance osteoblast and osteoclast formation. Proc Natl Acad Sci U S A 2006;103:14925-30.

9. Prior JC. FSH and bone-important physiology or not? Trends Mol Med 2007;13:1-3.

10. Zhu LL, Blair H, Cao J, Yuen T, Latif R, Guo L, et al. Blocking antibody to the $\beta$-subunit of FSH prevents bone loss by inhibiting bone resorption and stimulating bone synthesis. Proc Natl Acad Sci U S A 2012;109:14574-9.

11. Kara E, Dupuy L, Bouillon C, Casteret S, Maurel MC. Modulation of gonadotropins activity by antibodies. Front Endocrinol 2019; 10:15.

12. Cannon JG, Kraj B, Sloan G. Follicle-stimulating hormone promotes RANK expression on human monocytes. Cytokine 2011; 53:141-4.

13. Ji Y, Liu P, Yuen T, Haider S, He J, Romero R, et al. Epitope-specific monoclonal antibodies to FSHbeta increase bone mass. Proc Natl Acad Sci U S A 2018;115:2192-7.

14. Gass ML, Kagan R, Kohles JD, Martens MG. Bone turnover marker profile in relation to the menstrual cycle of premenopausal healthy women. Menopause 2008;15:667-75.

15. Gorai I, Taguchi Y, Chaki O, Kikuchi R, Nakayama M, Yang BC, et al. Serum soluble interleukin-6 receptor and biochemical markers of bone metabolism show significant variations during the menstrual cycle. J Clin Endocrinol Metab 1998;83: 326-32.

16. Mozzanega B, Gizzo S, Bernardi D, Salmaso L, Patrelli TS, Mioni R, et al. Cyclic variations of bone resorption mediators and markers in the different phases of the menstrual cycle. J Bone Miner Metab 2013;31:461-7.

17. Chiu KM, Ju J, Mayes D, Bacchetti P, Weitz S, Arnau CD. Changes in bone resorption during the menstrual cycle. J Bone Miner Res 1999;14:609-15.

18. Kalyan S, Patel MS, Kingwell E, Côté HCF, Liu D, Prior JC. Competing factors link to bone health in polycystic ovary syndrome: chronic low-grade inflammation takes a toll. Sci Rep 2017;7:3432.

19. Omodei U, Mazziotti G, Donarini G, Gola M, Guella V, Pagani F, et al. Effects of recombinant follicle-stimulating hormone on bone turnover markers in infertile women undergoing in vitro fertilization procedure. J Clin Endocrinol Metab 2013;98: 330-6.

20. Ecochard R, Guillerm A, Leiva R, Bouchard T, Direito A, Boehringer $\mathrm{H}$. Characterization of follicle stimulating hormone profiles in normal ovulating women. Fertil Steril 2014;102:237-43 e5.

21. Chubb SA. Measurement of C-terminal telopeptide of type I collagen (CTX) in serum. Clin Biochem 2012;45:928-35. 